\title{
Dominator Coloring Number of Middle Graph
}

\author{
T.Ramachandran ${ }^{1}$, A.Naseer Ahmed ${ }^{2}$ \\ ${ }^{1}$ (Department of Mathematics, M.V.M Government Arts college for Women, India) \\ ${ }^{2}$ (Department of Mathematics, Chettinad College of Engineering and Technology, India)
}

\begin{abstract}
Given a graph $G$, the dominator coloring problem seeks a proper coloring of $G$ with the additional property that every vertex in the graph dominates an entire color class. In this paper, as an extension of Dominator coloring some standard results for the middle graph of path and cycle has been discussed.
\end{abstract}

Keywords: Coloring, Domination, Dominator Coloring, Middle Graph

\section{Introduction}

In graph theory, coloring and dominating are two important areas which have been extensively studied. The fundamental parameter in the theory of graph coloring is the chromatic number $\chi(\mathrm{G})$ of a graph $\mathrm{G}$ which is defined to be the minimum number of colors required to color the vertices of $\mathrm{G}$ in such a way that no two adjacent vertices receive the same color. If $\chi(\mathrm{G})=\mathrm{k}$, we say that $\mathrm{G}$ is k-chromatic. A dominating set $\mathrm{S}$ is a subset of the vertices in a graph such that every vertex in the graph either belongs to $\mathrm{S}$ or has a neighbor in $\mathrm{S}$. The domination number is the order of a minimum dominating set. Given a graph $\mathrm{G}$ and an integer $\mathrm{k}$, finding a dominating set of order $\mathrm{k}$ is NP-complete on arbitrary graphs. [4,5] A graph has a dominator coloring if it has a proper coloring in which each vertex of the graph dominates every vertex of some color class. The dominator chromatic number $\chi_{\mathrm{d}}(\mathrm{G})$ is the minimum number of color classes in a dominator coloring of a graph $\mathrm{G}$. A $\chi_{\mathrm{d}}(\mathrm{G})$ - coloring of $\mathrm{G}$ is any dominator coloring with $\chi_{\mathrm{d}}(\mathrm{G})$ colors. Our study of this problem is motivated by [2] and [3].

\section{Terminologies}

We start with notation and more formal definitions.

Let $G=(V(G), E(G))$ be a graph with $n=|V(G)|$ and $m=|E(G)|$. For any vertex $v \in V(G)$, the open neighborhood of $\mathrm{v}$ is the set $\mathrm{N}(\mathrm{v})=\{\mathrm{u} \mid \mathrm{uv} \in \mathrm{E}(\mathrm{G})\}$ and the closed neighborhood is the set $\mathrm{N}[\mathrm{v}]=\mathrm{N}(\mathrm{v}) \cup \mathrm{v}$. Similarly, for any set $S \subseteq V(G), N(S)=\cup_{v \in S} N(v)-S$ and $N[S]=N(S) \cup S$. A set $S$ is a dominating set if $N[S]$ $=\mathrm{V}(\mathrm{G})$. The minimum cardinality of a dominating set of $\mathrm{G}$ is denoted by $\gamma(\mathrm{G})$.

The distance, $d(u, v)$, between two vertices $u$ and $v$ in $G$ is the smallest number of edges on a path between $u$ and $v$ in $G$. The eccentricity, $e(v)$, of a vertex $v$ is the largest distance from $v$ to any vertex of $G$. The radius, $\operatorname{rad}(\mathrm{G})$, of $\mathrm{G}$ is the smallest eccentricity in $\mathrm{G}$. The diameter, $\operatorname{diam}(\mathrm{G})$, of $\mathrm{G}$ is the largest eccentricity in G. A graph coloring is a mapping $\mathrm{f}: \mathrm{V}(\mathrm{G}) \rightarrow \mathrm{C}$, where $\mathrm{C}$ is a set of colors. A coloring $\mathrm{f}$ is proper if, for all $\mathrm{x}$. $\mathrm{y}$ $\in \mathrm{V}(\mathrm{G}), \mathrm{x} \in \mathrm{N}(\mathrm{y})$ implies $\mathrm{f}(\mathrm{x}) \neq \mathrm{f}(\mathrm{y})$. A k-coloring of $\mathrm{G}$ is a coloring that uses at most $\mathrm{k}$ colors. The chromatic number of $\mathrm{G}$ is $\chi(\mathrm{G})=\min \{\mathrm{k} \mid \mathrm{G}$ has a proper $\mathrm{k}$-coloring $\}$.

A coloring of $G$ can also be thought of as a partition of $V(G)$ into color classes $V_{1}, V_{2}, \ldots, V_{q}$, and a proper coloring of $\mathrm{G}$ is then a coloring in which each $\mathrm{Vi}, 1 \leq \mathrm{i} \leq \mathrm{q}$ is an independent set of $\mathrm{G}$, i.e., for each $\mathrm{i}$, the sub graph of G induced by Vi contains no edges. Dominator colorings were introduced in [6] and they were motivated by [2].

Definition 2.1: The Middle graph of $G$, denoted by $M(G)$ is defined as follows.

The vertex set of $M(G)$ is $V(G) \cup E(G)$. Two vertices $x$, $y$ in the vertex set of $M(G)$ are adjacent in $M(G)$ in case one of the following holds.

1. $x, y$ are in $E(G)$ and $x, y$ are adjacent in $G$.

2. $x$ is in $V(G)$, $y$ is in $E(G)$ and $x, y$ are incident in $G$ [7].

\section{Propositions 2.2:}

(1) The star $\mathrm{K}_{1, \mathrm{n}}$ has $\chi_{\mathrm{d}}\left(\mathrm{K}_{1, \mathrm{n}}\right)=2$.

(2) The path $\mathrm{P}_{\mathrm{n}}$ of order $\mathrm{n} \geq 3$ has $\chi_{d}\left(P_{n}\right)=\left\{\begin{array}{lr}1+\left\lceil\frac{\mathrm{n}}{3}\right\rceil & \text { if } \mathrm{n}=2,3,4,5,7 \\ 2+\left\lceil\frac{\mathrm{n}}{3}\right] & \text { otherwise }\end{array}\right.$

(3) The complete graph $K_{n}$ has and $\chi_{d}\left(K_{n}\right)=n$. 
(4) The cycle $\mathrm{C}_{\mathrm{n}}$ has $\chi_{d}\left(C_{n}\right)= \begin{cases}{\left[\frac{\mathrm{n}}{3}\right]} & \text { if } \mathrm{n}=4 \\ {\left[\frac{\mathrm{n}}{3}\right]+1} & \text { if } \mathrm{n}=5 \\ \left\lceil\frac{\mathrm{n}}{3}\right]+2 \text { otherwise }\end{cases}$

(5) The multi-star $K_{n}\left(a_{1}, a_{2}, \ldots, a_{n}\right)$ has $\chi_{d}\left(K_{n}\left(a_{1}, a_{2}, \ldots, a_{n}\right)\right)=n+1$

(6) The wheel $\mathrm{W}_{1, \mathrm{n}}$ has $\chi_{d}\left(\mathrm{~W}_{1, \mathrm{n}}\right)= \begin{cases}3 & \text { if } \mathrm{n} \text { is even } \\ 4 & \text { if } \mathrm{n} \text { is odd }\end{cases}$

(7) The complete k-partite graph $\boldsymbol{K}_{a_{1}, a_{2} \omega_{i} a_{k}}$ has $\chi_{d}\left(\boldsymbol{K}_{a_{1}, a_{2} a_{i} a_{k}}\right)=\boldsymbol{k}$

\section{Main Results}

Lemma: 3.1 Let $\mathrm{G}$ be a connected graph. Then $\max \{\chi(\mathrm{G}), \gamma(\mathrm{G})\} \leq \chi_{\mathrm{d}}(\mathrm{G}) \leq \chi(\mathrm{G})+\gamma(\mathrm{G})$. The bounds are sharp.

Lemma: 3.2 For any graph $\mathrm{G}, \chi(\mathrm{G}) \leq \chi_{\mathrm{d}}(\mathrm{G})$.

Lemma 3.3 Let $\mathrm{G}$ be a graph such that, $\mathrm{G}=\mathrm{M}\left(\mathrm{C}_{\mathrm{n}}\right)$ with $\mathrm{n}>3$, then $\chi_{\mathrm{d}}(\mathrm{G})=\frac{n+1}{2}+2$ for odd $\mathrm{n}$ and $\chi_{\mathrm{d}}(\mathrm{G})=\frac{n}{2}+2$ for even $n$

Proof: Let $C_{n}: v_{1}, v_{2}, \ldots . . v_{n}, v_{n+1}\left(=v_{1}\right)$ be a path of length $n$ and let $v_{i} v_{i+1}=e_{i}$ for $i=1,2 \ldots . n-1$ and $v_{1} v_{n}=e_{n}$. By the definition of middle graph, $M\left(C_{n}\right)$ has the vertex set $V\left(C_{n}\right) \times E\left(C_{n}\right)=\left\{v_{i} \mid 1 \leq i \leq n\right\} \cup\left\{e_{i} \mid 1 \leq i \leq n\right\}$ in which each $v_{i}$ is adjacent to $e_{i}$ and $e_{i-1}$ and each $e_{i}$ is adjacent to $v_{i+1}$ and $v_{i}$ for $i=2,3 \ldots \ldots n-1$ and $v_{1}$ is adjacent to $e_{1}$ and $e_{n}$, also $e_{n}$ is adjacent to $v_{1}$ and $v_{n}$. And for odd $n$, The minimal dominator color class partition is given by, $\left\{\left\{\mathrm{e}_{1}\right\},\left\{\mathrm{e}_{3}\right\}, \ldots,\left\{\mathrm{e}_{\mathrm{n}}\right\},\left\{\mathrm{e}_{2}, \mathrm{e}_{4}, \ldots, \mathrm{e}_{\mathrm{n}-1}\right\},\left\{\mathrm{v}_{1}, \mathrm{v}_{2}, \ldots, \mathrm{v}_{\mathrm{n}}\right\}\right\}$ for odd $\mathrm{n}$. And each vertex in color class partition dominates atleast one color class. Hence $\chi_{\mathrm{d}}(\mathrm{G})=\frac{n+1}{2}+1+1=\frac{n+1}{2}+2$. And for even $\mathrm{n}$, The minimal dominator color class partition is given by, $\left\{\left\{\mathrm{e}_{1}\right\},\left\{\mathrm{e}_{3}\right\} \ldots\left\{\mathrm{e}_{\mathrm{n}-1}\right\},\left\{\mathrm{e}_{2}, \mathrm{e}_{4}, \ldots, \mathrm{e}_{\mathrm{n}}\right\},\left\{\mathrm{v}_{1}, \mathrm{v}_{2}, \ldots, \mathrm{v}_{\mathrm{n}}\right\}\right\}$ for even $\mathrm{n}$.And each vertex in color class partition dominates atleast one color class. Hence $\chi_{\mathrm{d}}(\mathrm{G})=\frac{n}{2}+1+1=\frac{n}{2}+2$

Theorem 3.4 Let $\mathrm{G}$ be a graph such that, $\mathrm{G}=\mathrm{M}\left(\mathrm{C}_{\mathrm{n}}\right)$ with $\mathrm{n}>3$, then $\chi_{\mathrm{d}}(\mathrm{G})=\chi(\mathrm{G})+\gamma(\mathrm{G})-2$, for odd $\mathrm{n}$ and $\chi_{\mathrm{d}}$ $(\mathrm{G})=\chi(\mathrm{G})+\gamma(\mathrm{G})-1$, for even $\mathrm{n}$.

Proof: Let $\mathrm{G}$ be a graph such that, $\mathrm{G}=\mathrm{M}\left(\mathrm{C}_{\mathrm{n}}\right)$ with $\mathrm{n}>3$

For odd $\mathrm{n}$, clearly $\gamma(\mathrm{G})=\frac{n+1}{2}$ and $\chi(\mathrm{G})=4$.

Now, $\chi(\mathrm{G})+\gamma(\mathrm{G})-2=4+\frac{n+1}{2}-2$

$$
=\frac{n+1}{2}+2 \text {. }
$$

And also by lemma $3.3 \chi_{\mathrm{d}}(\mathrm{G})=\frac{n+1}{2}+2$

For even n, clearly $\gamma(\mathrm{G})=\frac{n}{2}$ and $\chi(\mathrm{G})=3$.

Now, $\chi(\mathrm{G})+\gamma(\mathrm{G})-1=3+\frac{n}{2}-1$

$$
=\frac{n}{2}+2
$$

And also by lemma $3.3 \chi_{\mathrm{d}}(\mathrm{G})=\frac{n}{2}+2$.

Lemma 3.5 Let $\mathrm{G}$ be a graph such that, $\mathrm{G}=\mathrm{M}\left(\mathrm{P}_{\mathrm{n}}\right)$ with $\mathrm{n}>2$, then $\chi_{\mathrm{d}}(\mathrm{G})=\frac{n+1}{2}+2$ for odd $\mathrm{n}$ and $\chi_{\mathrm{d}}(\mathrm{G})=\frac{n}{2}+3$, for even $n$.

Proof: Let $P_{n}: v_{1}, v_{2} \ldots \ldots . . . v_{n+1}$ be a path of length $n$ and let $v_{i} v_{i+1}=e_{i}$. By the definition of middle graph, $M\left(P_{n}\right)$ has the vertex set $V\left(P_{n}\right) \times E\left(P_{n}\right)=\left\{v_{i} \mid 1 \leq i \leq n+1\right\} \cup\left\{e_{i} \mid 1 \leq i \leq n\right\}$ in which each $v_{i}$ is adjacent to $e_{i}$ and $e_{i}$ is adjacent to $v_{i+1}$. Also $e_{i}$ is adjacent to $e_{i+1}$.

Case 1: If $\mathrm{n}$ is even. 
The minimal dominator color class partition is given by, $\left\{\left\{\mathrm{e}_{1}\right\},\left\{\mathrm{e}_{3}\right\}, \ldots,\left\{\mathrm{e}_{\mathrm{n}-1}\right\},\left\{\mathrm{e}_{2}, \mathrm{e}_{4}, \ldots, \mathrm{e}_{\mathrm{n}}\right\},\left\{\mathrm{v}_{1}, \mathrm{v}_{2}, \ldots, \mathrm{v}_{\mathrm{n}}\right.\right.$ \}$\left.\left\{\mathrm{v}_{\mathrm{n}+1}\right\}\right\}$. And each vertex in color class partition dominates atleast one color class.

Hence $\chi_{\mathrm{d}}(\mathrm{G})=\frac{n}{2}+1+1+1=\frac{n}{2}+3$

Case 2: If $n$ is odd.

The minimal dominator color class partition is given by, $\left\{\left\{\mathrm{e}_{1}\right\},\left\{\mathrm{e}_{3}\right\}, \ldots,\left\{\mathrm{e}_{\mathrm{n}}\right\},\left\{\mathrm{e}_{2}, \mathrm{e}_{4}, \ldots, \mathrm{e}_{\mathrm{n}-1}\right\},\left\{\mathrm{v}_{1}, \mathrm{v}_{2}, \ldots, \mathrm{v}_{\mathrm{n}}\right.\right.$ \}).And each vertex in color class partition dominates atleast one color class.

Hence $\chi_{\mathrm{d}}(\mathrm{G})=\frac{n+1}{2}+1+1=\frac{n+1}{2}+2$

Theorem 3.6 Let $\mathrm{G}$ be a graph such that, $\mathrm{G}=\mathrm{M}\left(\mathrm{P}_{\mathrm{n}}\right)$ with $\mathrm{n}>2$, then $\chi_{\mathrm{d}}(\mathrm{G})=\chi(\mathrm{G})+\gamma(\mathrm{G})-1$

Proof: Let $\mathrm{G}$ be a graph such that, $\mathrm{G}=\mathrm{M}\left(\mathrm{P}_{\mathrm{n}}\right)$ with $\mathrm{n}>2$.

For odd $n$, clearly $\gamma(\mathrm{G})=\frac{n+1}{2}$ and $\chi(\mathrm{G})=3$.

Now, $\chi(\mathrm{G})+\gamma(\mathrm{G})-1=3+\frac{n+1}{2}-1$

$$
=\frac{n+1}{2}+2
$$

And also by lemma $3.5 \chi_{\mathrm{d}}(\mathrm{G})=\frac{n+1}{2}+1$

For even $n$, clearly $\gamma(\mathrm{G})=\frac{n}{2}+1$ and $\chi(\mathrm{G})=3$.

Now, $\chi(\mathrm{G})+\gamma(\mathrm{G})-1=3+\frac{n}{2}+1-1$

$$
=\frac{n}{2}+3
$$

And also by lemma $3.5 \chi_{\mathrm{d}}(\mathrm{G})=\frac{n}{2}+3$.

\section{References}

[1]. Ralucca Michelle Gera, On Dominator Colorings in Graphs, Department of Applied Mathematics, Naval Postgraduate School, Monterey, CA 93943, USA

[2]. E.Cockayne, S. Hedetniemi, and S. Hedetniemi, Dominating partitions of graphs, tech. rep., 1979, unpublished manuscript

[3]. S. M. Hedetniemi, S. T. Hedetniemi, and A. A. McRae, Dominator colorings of graphs (2006) In preparation

[4]. T. W. Haynes, S. T. Hedetniemi, and P. J. Slater, Domination in Graphs: Advanced Topics, Marcel Dekker, New York, 1998

[5]. Marcel Dekker, Fundamentals of Domination in Graphs, New York, 1998

[6]. R. Gera, S Horton, C. Rasmussen, Dominator Colorings and Safe Clique Partitions, Congressus Numerantium (2006)

[7]. E.Sampathkumar and L. Pushpa Latha, Strong, weak Domination and Domination Balance in a Graph, Discrete Math. 161 (1996) 235-242. 